\title{
SEMI-CLASSICAL TIME-FREQUENCY ANALYSIS AND APPLICATIONS
}

\author{
ELENA CORDERO, MAURICE DE GOSSON, AND FABIO NICOLA
}

\begin{abstract}
This work represents a first systematic attempt to create a common ground for semi-classical and time-frequency analysis. These two different areas combined together provide interesting outcomes in terms of Schrödinger type equations. Indeed, continuity results of both Schrödinger propagators and their asymptotic solutions are obtained on $\hbar$-dependent Banach spaces, the semiclassical version of the well-known modulation spaces. Moreover, their operator norm is controlled by a constant independent of the Planck's constant $\hbar$. The main tool in our investigation is the joint application of standard approximation techniques from semi-classical analysis and a generalized version of Gabor frames, dependent of the parameter $\hbar$. Continuity properties of more general Fourier integral operators (FIOs) and their sparsity are also investigated.
\end{abstract}

\section{INTRODUCTION}

This paper represents a first systematic attempt to set up a joint framework for semi-classical and time-frequency analysis. There are many excellent contributions on wave packet decompositions in semi-classical analysis (cf., e.g., [3, 4, 22, 23, 31] and references therein). The main motivation for this topic is quantum mechanics: the basic theme is to understand the relationships between dynamical systems and the behavior of solutions to Schrödinger equations with a small positive parameter $\hbar \in(0,1]$, the Planck's constant, in other words, how classical mechanics is a limit of quantum mechanics. The main tool for this understanding is the use of coherent states. According to Gilmore-Perelomov [16], a coherent state system is an orbit for an irreducible group action in an Hilbert space. In particular, the most well-known coherent states are obtained by the Weyl-Heisenberg group action in $L^{2}\left(\mathbb{R}^{n}\right)$ and the standard Gaussian

$$
\psi_{0}(x)=2^{n / 4} e^{-\pi|x|^{2}} .
$$

2010 Mathematics Subject Classification. 42B35, 42C15, 47G30, 81Q20.

Key words and phrases. Time-frequency analysis, semi-classical analysis, Gabor frames, modulation spaces, Schrödinger equation, quadratic potentials. 
Recall that the $\hbar$-Weyl quantization of a function $H$ on the phase space $\mathbb{R}^{2 n}$ is formally defined by

$$
\begin{aligned}
\widehat{H} f(x)=\mathrm{Op}_{\hbar}^{w}[H] f(x) & =(2 \pi \hbar)^{-n} \int_{\mathbb{R}^{2 n}} e^{i \hbar^{-1}(x-y) \xi} H\left(\frac{x+y}{2}, \xi\right) f(y) d y d \xi \\
& =(2 \pi)^{-n} \int_{\mathbb{R}^{2 n}} e^{i(x-y) \xi} H\left(\frac{x+y}{2}, \hbar \xi\right) f(y) d y d \xi
\end{aligned}
$$

with $f$ in the Schwartz space $\mathcal{S}\left(\mathbb{R}^{n}\right)$. The function $H$ is called the $\hbar$-Weyl symbol of $\widehat{H}$. The Weyl-Heisenberg group action can be expressed by the Weyl quatization as follows. For $z_{0}=\left(x_{0}, p_{0}\right) \in \mathbb{R}^{2 n}, f \in \mathcal{S}\left(\mathbb{R}^{n}\right)$, the Weyl-Heisenberg operator $\widehat{\mathcal{T}}^{\hbar}\left(z_{0}\right)$ is given by

$$
\widehat{\mathcal{T}}^{\hbar}\left(z_{0}\right) f(x)=O p_{\hbar}^{w}\left[e^{i \hbar^{-1}\left(p_{0} x-x_{0} p\right)}\right] f(x)=e^{i \hbar^{-1}\left(p_{0} x-x_{0} p_{0} / 2\right)} f\left(x-x_{0}\right) .
$$

If $f=\psi_{0}$ in (11), $\widehat{\mathcal{T}}^{\hbar}\left(z_{0}\right) \psi_{0}, z_{0} \in \mathbb{R}^{2 n}$, are the canonical coherent states.

It is then not surprising that semi-classical analysis and time-frequency analysis are closely related because coherent states are the building blocks for the so-called $\hbar$ Gabor frames, an extension of Gabor frames, the bricks of time-frequency analysis.

Gabor frames or Weyl-Heisenberg frames (the latter terminology was introduced in [13]), are applied in several different areas: for characterization of smoothness properties, in particular for the definition of modulation spaces (cf. Section 2), for characterization of pseudodifferential operators and more generally Fourier integral operators (FIOs) (see Section 3) and of course in signal processing (cf. [18, 19] and references therein). They are widely employed in numerical analysis and used by engineers, but quite unknown among theoretical physicists. Although Gabor frames have been widely employed in the study of Schrödinger equations by two of us in the works [7, 8, 9, 11, 12], starting from the pioneering paper [10], there have not been displayed any direct connection with theoretical physics since the work of one of us [15]. In that paper the definition of semi-classical Gabor frames, named $\hbar$-Gabor frames, first appears.

Definition 1.1. Given a lattice $\Lambda$ in $\mathbb{R}^{2 n}$ and a non-zero function $g \in L^{2}\left(\mathbb{R}^{n}\right)$, the system

$$
\mathcal{G}^{\hbar}(g, \Lambda)=\left\{\widehat{\mathcal{T}}^{\hbar}(z) g: z \in \Lambda\right\}
$$

is called a semi-classical Gabor frame or $\hbar$-Gabor frame if it is a frame for $L^{2}\left(\mathbb{R}^{n}\right)$, that is there exist constants $0<A \leq B$ such that

$$
A\|f\|_{2}^{2} \leq \sum_{z \in \Lambda}\left|\left\langle f, \widehat{\mathcal{T}}^{\hbar}(z) g\right\rangle\right|^{2} \leq B\|f\|_{2}^{2}, \quad \forall f \in L^{2}\left(\mathbb{R}^{n}\right) .
$$

In particular, when $\hbar=(2 \pi)^{-1}$, we define

$$
\widehat{\mathcal{T}}(z)=\widehat{\mathcal{T}}^{(2 \pi)^{-1}}(z)
$$


and we recapture the standard definition of a Gabor frame simply by replacing the latter operator in (5) (see Section 2 for details).

In this work we present the main features of semi-classical Gabor frames, continuing their investigation started in [1, 15] (see also [25] for an application to functional integration).

The most well-known and used Banach spaces in time-frequency analysis are the so-called modulation spaces and Wiener amalgam spaces [17]. Here we focus on the former spaces, whose norm can be interpreted as a measure of the joint timefrequency distribution of a signal $f$ in $\mathcal{S}^{\prime}\left(\mathbb{R}^{n}\right)$, the space of tempered distributions. We refer to Section 2 for their exact definition and we recall that they are a scale of spaces comprehending the Sobolev spaces $H^{s}\left(\mathbb{R}^{n}\right)$, the Shubin-Sobolev spaces $\mathcal{Q}_{s}\left[2,29\right.$, hence, in particular, the Hilbert space $L^{2}\left(\mathbb{R}^{n}\right)$. For $\lambda>0$, we define the metaplectic operator $\widehat{D}_{\lambda}$ by

$$
\widehat{D}_{\lambda} f(x)=\lambda^{n / 2} f(\lambda x), \quad f \in L^{2}\left(\mathbb{R}^{n}\right) .
$$

If $M_{m}^{p, q}\left(\mathbb{R}^{n}\right)$ denotes the standard modulation space defined in Subsection 2.2, and

$$
h=2 \pi \hbar
$$

we propose

$$
M_{m}^{p, q, \hbar}\left(\mathbb{R}^{n}\right)=\widehat{D}_{h^{-1 / 2}} M_{m}^{p, q}\left(\mathbb{R}^{n}\right)
$$

as the semi-classical version of the space $M_{m}^{p, q}\left(\mathbb{R}^{n}\right)$. The main motivation is that these spaces turn out to be the right Banach spaces for continuity results of both exact and asymptotic solutions to semi-classical Schrödinger equations. Indeed we obtain norm estimates for such operators, uniformly with respect to the constant $\hbar$. Namely, our focus is the Cauchy problem in the semi-classical regime $\left(\hbar \rightarrow 0^{+}\right)$

$$
\left\{\begin{array}{l}
i \hbar \partial_{t} u=\widehat{H(t)} u \\
u(s)=u_{0},
\end{array}\right.
$$

where $t \in[0, T], u_{0} \in L^{2}\left(\mathbb{R}^{n}\right), s \in[0, T]$ is the initial time, and the quantum Hamiltonian $\widehat{H(t)}$ is supposed to be the $\hbar$-Weyl quantization of the classical observable $H(t, z)$, with $z=(x, \xi) \in \mathbb{R}^{2 n}$. Such a symbol is supposed to satisfy the following hypothesis:

Assumption $(\mathbf{H})$. The observable $H(t, z)$ is continuous with respect to $(t, z) \in$ $[0, T] \times \mathbb{R}^{2 n}$ and smooth in $z$, satisfying

$$
\left|\partial_{z}^{\alpha} H(t, z)\right| \leq C_{\alpha}, \quad \forall|\alpha| \geq 2, z \in \mathbb{R}^{2 n}, t \in[0, T] .
$$

We decompose the initial datum $u_{0}$ in (8) by means of a $\hbar$-Gabor frame whose atoms are Gaussian coherent states, construct asymptotic solutions for each of 
them, so-called Gaussian beams, and finally by superposition obtain the asymptotic solution (parametrix) to (8).

Note that in our construction of the parametrix to (8) we partially exploit the well-known tool of semi-classical approximation by Taylor series [3], already used in [1]: the approximate solution is searched as a finite sum of powers of $\hbar$, and the order of approximation can be arbitrarily large. Let us underline that here timefrequency analysis comes in to play by using $\hbar$-Gabor frames as coherent states. By combining tools from both semi-classical and time-frequency analysis (the latter developed in Subsection 2.1), we attain the desired continuity results (uniform with respect to $\hbar$ ) for the approximate solution on the semi-classical modulation spaces $M_{m}^{p, q, \hbar}$. Moreover, we present precise estimates of the error term in such spaces, again uniform with respect to $\hbar$, see Theorem 4.1 below, that can be regarded as the main result of this work.

All these issues let us claim that semi-classical Gabor frames and modulation spaces are, beyond $L^{2}$, the right Banach spaces to be used in quantum mechanics. This assertion is confirmed by the study of a broader class of Schrödinger-type propagators, the $\hbar$-dependent Fourier integral operators $\widehat{A}^{\hbar}: \mathcal{S}\left(\mathbb{R}^{n}\right) \rightarrow \mathcal{S}^{\prime}\left(\mathbb{R}^{n}\right)$ in the class $F I O_{\hbar}(\chi, s)$, defined in terms of the decay properties of the entries of their so-called $\hbar$-Gabor matrix

$$
\left|\left\langle\widehat{A}^{\hbar} \mathcal{T}^{\hbar}(z) g^{h}, \mathcal{T}^{\hbar}(w) g^{h}\right\rangle\right| \leq \frac{C_{s}}{\left\langle h^{-1 / 2}(w-\chi(z))\right\rangle^{s}}, \quad z, w \in \mathbb{R}^{2 n}
$$

where $\langle\cdot\rangle=\left(1+|\cdot|^{2}\right)^{1 / 2}, \chi$ is a tame symplectomorphism (introduced in [10], see also [6]) and $C_{s}>0$ is independent of $\hbar$; see Definition 3.1 below.

In the following Section 3 we investigate the main properties of such operators. In particular, we furnish again continuity properties on semi-classical modulation spaces and the so-called sparsity properties of such operators. Roughly speaking, there are finitely many entries in (10) that are not negligible, so that such operators can be efficiently represented numerically.

This paper can be regarded as a first step of a project aiming at allowing semiclassical and time-frequency analysis to talk to each other. We believe that joining the main features of these disciplines will provide an advancement in the understanding of both areas.

The work is organized as follows: Section 2 contains the preliminary notions from time-frequency analysis and the study of semi-classical Gabor frames and modulation spaces. In Section 3 we study the main properties of semi-classical Fourier integral operators and provide an application to Schrödinger propagators. Section 4 contains the parametrix construction for Schrödinger equations and exhibits the main result of this paper: Theorem 4.1. 


\section{Preliminaries AND time-FREQUenCy ANALYSis tools}

The metaplectic group is denoted by $M p(n)$. Consider $\widehat{S} \in M p(n)$ with covering projection $\pi^{\hbar}: \widehat{S} \mapsto S \in \mathrm{Sp}(n, \mathbb{R})$ the symplectic group of real $2 n \times 2 n$ matrices. The appearance of the subscript $\hbar$ is due to the fact that to the $\hbar$-dependent operator $\widehat{V}_{P} f(x)=e^{-i P x \cdot x /(2 \hbar)} f(x)$ (chirp) corresponds the projection $\pi^{\hbar}\left(\widehat{V}_{P}\right)=$ $V_{P}$, with $V_{P}=\left(\begin{array}{cc}I_{n} & 0_{n} \\ -P & 0_{n}\end{array}\right), P=P^{T}$, and to the Fourier transform $\widehat{J} f(x)=$ $(2 \pi i \hbar)^{-n / 2} \int_{\mathbb{R}^{n}} e^{-i x x^{\prime} / \hbar} f\left(x^{\prime}\right) d x^{\prime}$ corresponds $\pi^{\hbar}(\widehat{J})=J$, defined by

$$
J=\left(\begin{array}{cc}
0_{n} & I_{n} \\
-I_{n} & 0_{n}
\end{array}\right) .
$$

For details see [15, Appendix A] and the books [3, 14]. In particular, for $\lambda>0$ we shall use the metaplectic operator $\widehat{D}_{\lambda} \in M p(n)$ defined in (6) and whose projection is $\pi^{\hbar}\left(\widehat{D}_{\lambda}\right)=D_{\lambda}$, the symplectic matrix

$$
D_{\lambda}=\left(\begin{array}{cc}
\lambda^{-1} I_{n} & 0_{n} \\
0_{n} & \lambda I_{n}
\end{array}\right)
$$

In the sequel we shall often use the fundamental symplectic covariance formula

$$
\widehat{\mathcal{T}}^{\hbar}(z) \widehat{S}=\widehat{S} \widehat{\mathcal{T}}^{\hbar}\left(S^{-1} z\right) \quad S \in \operatorname{Sp}(n, \mathbb{R}) .
$$

2.1. Semi-classical Gabor frames. Consider a lattice $\Lambda$ in $\mathbb{R}^{2 n}$. For $g \in L^{2}\left(\mathbb{R}^{n}\right)$, the Gabor system

$$
\mathcal{G}(g, \Lambda)=\{\widehat{\mathcal{T}}(z) g, z \in \Lambda\}
$$

(recall that $\left.\widehat{\mathcal{T}}(z)=\widehat{\mathcal{T}}^{(2 \pi)^{-1}}(z)\right)$ is a Gabor frame for $L^{2}\left(\mathbb{R}^{n}\right)$ if there exist constants $A, B>0$ such that for every $f \in L^{2}\left(\mathbb{R}^{n}\right)$

$$
A\|f\|_{2}^{2} \leq \sum_{z \in \Lambda}|\langle f, \widehat{\mathcal{T}}(z) g\rangle|^{2} \leq B\|f\|_{2}^{2} .
$$

Observe that, up to a phase factor, $\widehat{\mathcal{T}}(z)$ is the so-called time-frequency (or phasespace) shift

$$
\widehat{\mathcal{T}}(z) f(t)=e^{-\pi i \xi x} e^{2 \pi i \xi t} f(t-x)=e^{-\pi i \xi x} M_{\xi} T_{x} f(t), \quad z=(x, \xi),
$$

where translation and modulation operators are defined by

$$
T_{x} f(t)=f(t-x) \quad \text { and } \quad M_{\xi} f(t)=e^{2 \pi i \xi t} f(t) .
$$

If (14) holds, then there exists a $\gamma \in L^{2}\left(\mathbb{R}^{n}\right)$ (so-called dual window), such that $\mathcal{G}(\gamma, \Lambda)$ is a frame for $L^{2}\left(\mathbb{R}^{n}\right)$ and every $f \in L^{2}\left(\mathbb{R}^{n}\right)$ can be expanded as

$$
f=\sum_{z \in \Lambda}\langle f, \widehat{\mathcal{T}}(z) g\rangle \widehat{\mathcal{T}}(z) \gamma=\sum_{z \in \Lambda}\langle f, \widehat{\mathcal{T}}(z) \gamma\rangle \widehat{\mathcal{T}}(z) g
$$


with unconditional convergence in $L^{2}\left(\mathbb{R}^{n}\right)$. For $\Lambda=\alpha \mathbb{Z}^{n} \times \beta \mathbb{Z}^{n}$, with $\alpha, \beta>0$, it was proved by Lyubarski [24], by Seip and Wallsten [27, 28] in dimension $n=1$, and then easily extended using tensor product arguments the frame property for the Gaussian function $\psi_{0}$ in (1). The result reads as follows. For $\Lambda=\alpha \mathbb{Z}^{n} \times \beta \mathbb{Z}^{n}$, we use the notation $\mathcal{G}(g, \alpha, \beta)$ instead of $\mathcal{G}(g, \Lambda)$ whenever it is more suitable.

Theorem 2.1. The system $\mathcal{G}\left(\psi_{0}, \alpha, \beta\right)$, where $\psi_{0}$ is the Gaussian function defined in (1), is a frame for $L^{2}\left(\mathbb{R}^{n}\right)$ if and only if $\alpha \beta<1$.

Observe that, for any $g \in L^{2}\left(\mathbb{R}^{n}\right), r>0$,

$$
\widehat{D}_{r^{-1 / 2}}\left(T_{\alpha m} M_{\beta n} g\right)=T_{\alpha r^{1 / 2} m} M_{\beta r^{-1 / 2}{ }_{n}} \widehat{D}_{r^{-1 / 2}} g
$$

hence the Gabor system $\mathcal{G}\left(\psi_{0}, \alpha, \beta\right)$ is mapped by $\widehat{D}_{r^{-1 / 2}}$ to the Gabor system $\mathcal{G}\left(\widehat{D}_{r^{-1 / 2}} \psi_{0}, \alpha r^{1 / 2}, \beta r^{-1 / 2}\right)$.

Since $\widehat{D}_{r^{-1 / 2}}$ is a bijective isometry of $L^{2}\left(\mathbb{R}^{n}\right), \mathcal{G}\left(\psi_{0}, \alpha, \beta\right)$ is a frame if and only if $\mathcal{G}\left(\widehat{D}_{r^{-1 / 2}} \psi_{0}, \alpha r^{1 / 2}, \beta r^{-1 / 2}\right)$ is, and the frame bounds coincide (in particular they are independent of $r$ ). For $r=2 \pi$, notice that $\widehat{D}_{(2 \pi)^{-1 / 2}} \psi_{0}=\phi_{0}$, where

$$
\phi_{0}(x)=\pi^{-n / 4} e^{-|x|^{2} / 2},
$$

so that $\mathcal{G}\left(\phi_{0}, \alpha(2 \pi)^{1 / 2}, \beta(2 \pi)^{-1 / 2}\right)$ is a Gabor frame if and only if $\alpha \beta<1$ and with the same frame bounds as $\mathcal{G}\left(\psi_{0}, \alpha, \beta\right)$.

We define its rescaled version by

$$
\phi_{0}^{\hbar}(x)=\widehat{D}_{\hbar^{-1 / 2}} \phi_{0}(x)=(\pi \hbar)^{-n / 4} e^{-|x|^{2} /(2 \hbar)} .
$$

Arguing as above we infer that $\mathcal{G}\left(\phi_{0}^{\hbar}, \alpha(2 \pi \hbar)^{1 / 2}, \beta(2 \pi \hbar)^{-1 / 2}\right)$ is a Gabor frame if and only if $\alpha \beta<1$ and the frame bounds coincide with those of $\mathcal{G}\left(\psi_{0}, \alpha, \beta\right)$. In particular, the frame bounds do not depend on the Plank constant $\hbar$. Indeed, defining $h$ as in (7), observe that

$$
\phi_{0}^{\hbar}=\widehat{D}_{h^{-1 / 2}} \psi_{0}
$$

hence the Gabor system $\mathcal{G}\left(\phi_{0}^{\hbar}, \alpha h^{1 / 2}, \beta h^{-1 / 2}\right)$ is the image of $\mathcal{G}\left(\psi_{0}, \alpha, \beta\right)$ under the dilation $\widehat{D}_{h^{-1 / 2}}$.

Let us also recall that the Gabor frame $\mathcal{G}\left(\psi_{0}, \alpha, \beta\right)$ admits a dual window $\gamma_{0}$ (that is not the canonical one) such that $\gamma_{0} \in \mathcal{S}\left(\mathbb{R}^{n}\right)$, cf. [21].

From now onward we use the notation

$$
g^{h}=\widehat{D}_{h^{-1 / 2}} g
$$

for any function $g \in L^{2}\left(\mathbb{R}^{n}\right)$, so that the rescaled version of the dual window $\gamma_{0}$ reads

$$
\gamma_{0}^{h}=\widehat{D}_{h^{-1 / 2}} \gamma_{0}
$$


The main properties of semi-classical Gabor frames, recalled in Definition 1.1 were investigated in [1, Proposition 2.3] (see also [15]). We recall Proposition 2.3 in [1], which shows how to switch from a $\hbar$-Gabor frame to a standard Gabor frame and vice-versa:

Proposition 2.2. The system $\mathcal{G}(\gamma, \Lambda)$ is a dual Gabor frame of $\mathcal{G}(g, \Lambda)$ if and only if $\mathcal{G}^{\hbar}\left(\gamma^{h}, h^{1 / 2} \Lambda\right)$ is a dual $\hbar$-Gabor frame of the $\hbar$-Gabor frame $\mathcal{G}^{\hbar}\left(g^{h}, h^{1 / 2} \Lambda\right)$. Moreover, the frame bounds of $\mathcal{G}(g, \Lambda)$ and $\mathcal{G}^{\hbar}\left(g^{h}, h^{1 / 2} \Lambda\right)$ coincide, and the same holds for their dual frames.

Using Proposition 2.2 for the Gaussian $\psi_{0}$ and its dual window $\gamma_{0} \in \mathcal{S}\left(\mathbb{R}^{n}\right)$, we can state:

Corollary 2.3. For $\Lambda=\alpha \mathbb{Z}^{n} \times \beta \mathbb{Z}^{n}, \alpha \beta<1$, the system $\mathcal{G}^{\hbar}\left(\gamma_{0}^{h}, h^{1 / 2} \Lambda\right)$ is a dual frame of the $\hbar$-Gabor frame $\mathcal{G}^{\hbar}\left(\phi_{0}^{\hbar}, h^{1 / 2} \Lambda\right)$. Moreover, the frame bounds of $\mathcal{G}^{\hbar}\left(\gamma_{0}^{h}, h^{1 / 2} \Lambda\right)$ are the same as those of $\mathcal{G}\left(\gamma_{0}, \Lambda\right)$. In particular, they are independent of $\hbar$.

2.2. Semi-classical Modulation Spaces. The Banach spaces under our consideration will be a semi-classical version of modulation spaces.

Modulation spaces were introduced by Feichtinger in [17] and have been widely employed over the last 20 years in the framework of time-frequency analysis. For their definition, we need to recall the notion of weight functions on the timefrequency plane, which intervene in the description of the decay properties of a function/distribution. We denote by $v$ a continuous, positive, even, submultiplicative weight function (in short, a submultiplicative weight), i.e., $v(0)=1$, $v(z)=v(-z)$, and $v\left(z_{1}+z_{2}\right) \leq v\left(z_{1}\right) v\left(z_{2}\right)$, for all $z, z_{1}, z_{2} \in \mathbb{R}^{2 n}$. A positive, even weight function $m$ on $\mathbb{R}^{2 n}$ is called $v$-moderate if $m\left(z_{1}+z_{2}\right) \leq C v\left(z_{1}\right) m\left(z_{2}\right)$ for all $z_{1}, z_{2} \in \mathbb{R}^{2 n}$. We denote by $\mathcal{M}_{v}$ the class of all $v$-moderate weights. In this paper we will mainly work with the polynomial weights

$$
v_{s}(z)=\langle z\rangle^{s}=\left(1+|z|^{2}\right)^{\frac{s}{2}}, \quad z \in \mathbb{R}^{2 n}, \quad s \in \mathbb{R} .
$$

Let $f \in \mathcal{S}^{\prime}\left(\mathbb{R}^{n}\right)$. For $z=(x, \xi) \in \mathbb{R}^{2 n}$, we define the short-time Fourier transform $(\mathrm{STFT})$ of $f$ as

$$
V_{g} f(z)=\left\langle f, M_{\xi} T_{x} g\right\rangle=\int_{\mathbb{R}^{n}} f(t) \overline{g(t-x)} e^{-2 \pi i t \xi} d t .
$$

Observe that

$$
\left|V_{g} f(z)\right|=|\langle f, \widehat{\mathcal{T}}(z) g\rangle|, \quad \forall z \in \mathbb{R}^{2 n} .
$$

Given a non-zero window $g \in \mathcal{S}\left(\mathbb{R}^{n}\right)$, a $v$-moderate weight function $m$ on $\mathbb{R}^{2 n}$, $1 \leq p, q \leq \infty$, the modulation space $M_{m}^{p, q}\left(\mathbb{R}^{n}\right)$ consists of all tempered distributions 
$f \in \mathcal{S}^{\prime}\left(\mathbb{R}^{n}\right)$ such that the STFT $V_{g} f$ is in $L_{m}^{p, q}\left(\mathbb{R}^{2 n}\right)$ (weighted mixed-norm spaces), with norm

$$
\|f\|_{M_{m}^{p, q}}=\left\|V_{g} f\right\|_{L_{m}^{p, q}}=\left(\int_{\mathbb{R}^{n}}\left(\int_{\mathbb{R}^{n}}\left|V_{g} f(x, \xi)\right|^{p} m(x, \xi)^{p} d x\right)^{q / p} d \xi\right)^{1 / q} .
$$

(Obvious modifications occur when $p=\infty$ or $q=\infty$ ). If $p=q$, we write $M_{m}^{p}\left(\mathbb{R}^{n}\right)$ instead of $M_{m}^{p, p}\left(\mathbb{R}^{n}\right)$, and if $m(z) \equiv 1$ on $\mathbb{R}^{2 n}$, then we write $M^{p, q}\left(\mathbb{R}^{n}\right)$ and $M^{p}\left(\mathbb{R}^{n}\right)$ for $M_{m}^{p, q}\left(\mathbb{R}^{n}\right)$ and $M_{m}^{p, p}\left(\mathbb{R}^{n}\right)$. Then $M_{m}^{p, q}\left(\mathbb{R}^{n}\right)$ is a Banach space whose definition is independent of the choice of the window $g$, in the sense that different non-zero window functions yield equivalent norms.

In the following we shall work with the a rescaled version of the Schwartz seminorms and modulation space norms, to make the corresponding spaces suitable for the analysis in the semi-classical regime, where we are looking for estimates independent of $\hbar$.

Namely, let us define, for $0<\hbar \leq 1$,

$$
\mathcal{S}^{\hbar}\left(\mathbb{R}^{n}\right)=\widehat{D}_{h^{-1 / 2}} \mathcal{S}\left(\mathbb{R}^{n}\right)=\left\{f \in \mathcal{C}^{\infty}\left(\mathbb{R}^{n}\right):\left\|\widehat{D}_{h^{1 / 2}} f\right\|_{k}<\infty\right\},
$$

where $\left\{\|\cdot\|_{k}\right\}_{k} k \in \mathbb{N}$, is the family of seminorms defining the Schwartz class $\mathcal{S}\left(\mathbb{R}^{n}\right)$ :

$$
\|f\|_{k}=\sum_{|\alpha| \leq k}\left\|\left(1+|\cdot|^{k}\right) \partial^{\alpha} f\right\|_{\infty} .
$$

Clearly $\mathcal{S}^{\hbar}\left(\mathbb{R}^{n}\right)=\mathcal{S}\left(\mathbb{R}^{n}\right)$ as sets.

Definition 2.4. For $m \in \mathcal{M}_{v}, 1 \leq p, q \leq \infty, 0<\hbar \leq 1$, we define

$$
M_{m}^{p, q, \hbar}\left(\mathbb{R}^{n}\right)=\widehat{D}_{h^{-1 / 2}} M_{m}^{p, q}\left(\mathbb{R}^{n}\right)=\left\{f \in \mathcal{S}^{\prime}\left(\mathbb{R}^{n}\right):\|f\|_{M_{m}^{p, q, \hbar}}:=\left\|\widehat{D}_{h^{1 / 2}} f\right\|_{M_{m}^{p, q}}<\infty\right\} .
$$

Using $\left(M_{m}^{p, q}\right)^{*}\left(\mathbb{R}^{n}\right)=M_{1 / m}^{p^{\prime}, q^{\prime}}\left(\mathbb{R}^{n}\right)$, for $1 \leq p, q<\infty$, we infer

$$
\left(M_{m}^{p, q, \hbar}\right)^{*}\left(\mathbb{R}^{n}\right)=M_{1 / m}^{p^{\prime}, q^{\prime}, 1 / \hbar}\left(\mathbb{R}^{n}\right)
$$

for $1 \leq p, q<\infty$.

Fix $g \in \mathcal{S}\left(\mathbb{R}^{n}\right) \backslash\{0\}$. For $f \in M_{m}^{p, q, \hbar}\left(\mathbb{R}^{n}\right)$, let us compute explicitly the STFT of $\widehat{D}_{h^{1 / 2}} f$. Writing $z=(x, \xi)$, using $\widehat{\mathcal{T}}(x, \xi)=\widehat{\mathcal{T}}^{\hbar}(x, 2 \pi \hbar \xi)$ and the covariance property $\widehat{D}_{h^{-1 / 2}} \widehat{\mathcal{T}}^{\hbar}(z)=\widehat{\mathcal{T}}^{\hbar}\left(D_{h^{-1 / 2}} z\right) \widehat{D}_{h^{-1 / 2}}$, we obtain

$$
\begin{aligned}
\left|V_{g}\left(\widehat{D}_{h^{1 / 2}} f\right)(x, \xi)\right| & =\left|\left\langle\widehat{D}_{h^{1 / 2}} f, \widehat{\mathcal{T}}(z) g\right\rangle\right|=\left|\left\langle\widehat{D}_{h^{1 / 2}} f, \widehat{\mathcal{T}}^{\hbar}(x, 2 \pi \hbar \xi) g\right\rangle\right| \\
& =\left|\left\langle f, \widehat{D}_{h^{-1 / 2}} \widehat{\mathcal{T}}^{\hbar}(x, 2 \pi \hbar \xi) g\right\rangle\right|=\left|\left\langle f, \widehat{\mathcal{T}}^{\hbar}\left(h^{1 / 2} x, h^{1 / 2} \xi\right) \widehat{D}_{h^{-1 / 2}} g\right\rangle\right| \\
& =\left|\left\langle f, \widehat{\mathcal{T}}^{\hbar}\left(h^{1 / 2} x, h^{1 / 2} \xi\right) g^{h}\right\rangle\right|
\end{aligned}
$$

(in the last row we used the notation (19)). The previous computations motivate the definition of a semi-classical version of the STFT as follows. 
Definition 2.5. Fix $g \in \mathcal{S}\left(\mathbb{R}^{n}\right) \backslash\{0\}$. We define the $\hbar$-short-time Fourier transform ( $\hbar$-STFT) $V_{g}^{\hbar} f$ of a function/distribution $f \in M_{m}^{p, q, \hbar}\left(\mathbb{R}^{n}\right)$ by

$$
V_{g}^{\hbar} f(z)=\left\langle f, \widehat{\mathcal{T}}^{\hbar}\left(h^{1 / 2} z\right) g^{h}\right\rangle, \quad \forall z \in \mathbb{R}^{2 n} .
$$

Fixed $g \in \mathcal{S}\left(\mathbb{R}^{n}\right) \backslash\{0\}$, taking $f \in M_{m}^{p, q, \hbar}\left(\mathbb{R}^{n}\right)$, we reckon

$$
\begin{aligned}
\|f\|_{M_{m}^{p, q, \hbar}} & =\left\|\widehat{D}_{h^{1 / 2}} f\right\|_{M_{m}^{p, q}}=\left(\int_{\mathbb{R}^{n}}\left(\int_{\mathbb{R}^{n}}\left|V_{g}\left(\widehat{D}_{h^{1 / 2}} f\right)(x, \xi)\right|^{p} m(x, \xi)^{p}\right)^{\frac{q}{p}} d \xi\right)^{\frac{1}{q}} \\
& =\left(\int_{\mathbb{R}^{n}}\left(\int_{\mathbb{R}^{n}}\left|\left\langle f, \widehat{\mathcal{T}}^{\hbar}\left(h^{1 / 2} x, h^{1 / 2} \xi\right) g^{h}\right\rangle\right|^{p} m(x, \xi)^{p}\right)^{\frac{q}{p}} d \xi\right)^{\frac{1}{q}} \\
& =\left(\int_{\mathbb{R}^{n}}\left(\int_{\mathbb{R}^{n}}\left|V_{g}^{\hbar} f\right|^{p} m(x, \xi)^{p}\right)^{\frac{q}{p}} d \xi\right)^{\frac{1}{q}} .
\end{aligned}
$$

Hence, an equivalent definition of the semi-classical modulation spaces is as follows: Fix $g \in \mathcal{S}\left(\mathbb{R}^{n}\right) \backslash\{0\}$, then

$$
M_{m}^{p, q, \hbar}\left(\mathbb{R}^{n}\right)=\left\{f \in \mathcal{S}^{\prime}\left(\mathbb{R}^{n}\right):\left\|V_{g}^{\hbar} f\right\|_{L_{m}^{p, q}}<\infty\right\} .
$$

As Gabor frames characterize modulation spaces, we shall show that $\hbar$-Gabor frames characterize semi-classical modulation spaces.

Given a lattice $\Lambda \subset \mathbb{R}^{2 n}$, a Gabor frame $\mathcal{G}(g, \Lambda)$ with dual window $\gamma \in L^{2}\left(\mathbb{R}^{n}\right)$, let us recall the coefficient (or analysis) operator $C_{g}: L^{2}\left(\mathbb{R}^{n}\right) \rightarrow \ell^{2}(\Lambda)$, given by

$$
C_{g} f=(\langle f, \widehat{\mathcal{T}}(z) g\rangle)_{z \in \Lambda}
$$

and the reconstruction (or synthesis) operator $D_{\gamma}: \ell^{2}(\Lambda) \rightarrow L^{2}\left(\mathbb{R}^{n}\right)$ :

$$
R_{\gamma} c=\sum_{z \in \Lambda} c_{z} \widehat{\mathcal{T}}(z) \gamma, \quad c \in \ell^{2}(\Lambda)
$$

The related Gabor frame operator is defined by

$$
S_{\gamma, g} f=\sum_{z \in \Lambda}\langle f, \widehat{\mathcal{T}}(z) g\rangle \widehat{\mathcal{T}}(z) \gamma
$$

The fact that $\gamma$ is a dual window of $g$ can be equivalently written as $S_{\gamma, g}=I$ on $L^{2}\left(\mathbb{R}^{n}\right)$, that is the Gabor frame operator is the identity operator on $L^{2}\left(\mathbb{R}^{n}\right)$. The semi-classical analysis $C_{g}^{\hbar}$, synthesis $R_{\gamma}^{\hbar}$ and frame operators $S_{\gamma, g}^{\hbar}$ (also called $\hbar$-analysis, $\hbar$-synthesis and $\hbar$-frame operators) are obtained simply by substituting $\widehat{\mathcal{T}}(z)$ with the Weyl-Heisenberg shift $\widehat{\mathcal{T}}^{\hbar}(z)$ :

$$
C_{g}^{\hbar} f=\left(\left\langle f, \widehat{\mathcal{T}}^{\hbar}(z) g\right\rangle\right)_{z \in \Lambda},
$$




$$
\begin{gathered}
R_{\gamma}^{\hbar} c=\sum_{z \in \Lambda} c_{z} \widehat{\mathcal{T}^{\hbar}}(z) \gamma, \quad c \in \ell^{2}(\Lambda) . \\
S_{\gamma, g}^{\hbar} f=\sum_{z \in \Lambda}\left\langle f, \widehat{\mathcal{T}}^{\hbar}(z) g\right\rangle \widehat{\mathcal{T}}^{\hbar}(z) \gamma .
\end{gathered}
$$

Provided the window $g, \gamma$ are smooth enough, we have the following characterization for modulation spaces (see, e.g. [20, Corollary 12.2.6]).

Proposition 2.6. Assume $g, \gamma \in M_{v}^{1}\left(\mathbb{R}^{n}\right)$ and that $S_{\gamma, g}=I$ on $L^{2}\left(\mathbb{R}^{n}\right)$. Then

$$
\begin{aligned}
f & =\sum_{k, n \in \mathbb{Z}^{n}}\left\langle f, T_{\alpha k} M_{\beta n} g\right\rangle T_{\alpha k} M_{\beta n} \gamma \\
& =\sum_{k, n \in \mathbb{Z}^{n}}\left\langle f, T_{\alpha k} M_{\beta n} \gamma\right\rangle T_{\alpha k} M_{\beta n} g
\end{aligned}
$$

with unconditional convergence in $M_{m}^{p, q}\left(\mathbb{R}^{n}\right)$ if $1 \leq p, q<\infty$ and weak $k^{*}$ convergence in $M_{1 / v}^{\infty}\left(\mathbb{R}^{n}\right)$ otherwise. Furthermore, there are constants $0<A \leq B$ such that

$$
A\|f\|_{M_{m}^{p, q}\left(\mathbb{R}^{n}\right)} \leq\left\|\left\langle f, T_{\alpha k} M_{\beta n} g\right\rangle\right\|_{\ell_{m}^{p, q}(\Lambda)} \leq B\|f\|_{M_{m}^{p, q}\left(\mathbb{R}^{n}\right)}, \forall f \in M_{m}^{p, q}\left(\mathbb{R}^{n}\right) .
$$

(Similar estimates hold by replacing $g$ with $\gamma$ ).

For a given weight function $m \in \mathcal{M}_{v}$, let us introduce the notation

$$
m_{\hbar}(z):=m\left(h^{-1 / 2} z\right)
$$

Then we obtain the following characterization for semi-classical modulation spaces.

Proposition 2.7. Under the assumptions of Proposition [2.6, we have:

(i) The $\hbar$-analysis operator $C_{g}^{\hbar}$ is bounded from $M_{m}^{p, q, \hbar}\left(\mathbb{R}^{n}\right)$ to $\ell_{m_{\hbar}}^{p, q}\left(h^{1 / 2} \Lambda\right)$.

(ii) The $\hbar$-synthesis operator $R_{\gamma}^{\hbar}$ is bounded from $\ell_{m_{\hbar}}^{p, q}\left(h^{1 / 2} \Lambda\right)$ to $M_{m}^{p, q, \hbar}\left(\mathbb{R}^{n}\right)$.

(iii) The $\hbar$-frame operator satisfies $S_{\gamma, g}^{\hbar}=I$ on $M_{m}^{p, q, \hbar}\left(\mathbb{R}^{n}\right)$. Namely,

$$
\begin{aligned}
f & =\sum_{z \in h^{1 / 2} \Lambda}\left\langle f, \widehat{\mathcal{T}}^{\hbar}(z) g^{h}\right\rangle \widehat{\mathcal{T}}^{\hbar}(z) \gamma^{h} \\
& =\sum_{z \in h^{1 / 2} \Lambda}\left\langle f, \widehat{\mathcal{T}}^{\hbar}(z) \gamma^{h}\right\rangle \widehat{\mathcal{T}}^{\hbar}(z) g^{h}
\end{aligned}
$$

with unconditional convergence in $M_{m}^{p, q, \hbar}\left(\mathbb{R}^{n}\right)$ if $1 \leq p, q<\infty$ and weak $k^{*}$ convergence in $M_{1 / v}^{\infty, 1 / \hbar}\left(\mathbb{R}^{n}\right)$ otherwise.

Furthermore, there are constants $0<A \leq B$, independent of $\hbar$, such that

$$
A\|f\|_{M_{m}^{p, q, \hbar}\left(\mathbb{R}^{n}\right)} \leq\left\|\left\langle f, \widehat{\mathcal{T}}^{\hbar}(z) g^{h}\right\rangle\right\|_{\ell_{m_{\hbar}}^{p, q}\left(h^{1 / 2} \Lambda\right)} \leq B\|f\|_{M_{m}^{p, q, \hbar}\left(\mathbb{R}^{n}\right)}, \quad \forall f \in M_{m}^{p, q, \hbar}\left(\mathbb{R}^{n}\right) .
$$

(Similar estimates hold by replacing $g^{h}$ with $\gamma^{h}$ ). 
Proof. Observe that the metaplectic operators $\widehat{D}_{h^{1 / 2}}$ is an isometric isomorphism from $M_{m}^{p, q, \hbar}\left(\mathbb{R}^{n}\right)$ to $M_{m}^{p, q}\left(\mathbb{R}^{n}\right)$ with inverse $\widehat{D}_{h^{-1 / 2}}$. We treat the case $p, q<\infty$, the other case is similar. By Proposition 2.6, for any $f \in M_{m}^{p, q, \hbar}\left(\mathbb{R}^{n}\right)$, we can write, for $z=(x, \xi)$, and using the covariance property $\widehat{D}_{h^{-1 / 2}} \widehat{\mathcal{T}}^{\hbar}(z)=\widehat{\mathcal{T}}^{\hbar}\left(D_{h^{-1 / 2}} z\right) \widehat{D}_{h^{-1 / 2}}$,

$$
\begin{aligned}
\widehat{D}_{h^{1 / 2}} f & =\sum_{z \in \Lambda}\left\langle\widehat{D}_{h^{1 / 2}} f, \widehat{\mathcal{T}}(z) g\right\rangle \widehat{\mathcal{T}}(z) \gamma \\
& =\sum_{z \in \Lambda}\left\langle f, \widehat{D}_{h^{-1 / 2}} \widehat{\mathcal{T}}(z) g\right\rangle \widehat{\mathcal{T}}(z) \gamma \\
& =\sum_{(x, \xi) \in \Lambda}\left\langle f, \widehat{D}_{h^{-1 / 2}} \widehat{\mathcal{T}}^{\hbar}(x, 2 \pi \hbar \xi) g\right\rangle \widehat{\mathcal{T}}^{\hbar}(x, 2 \pi \hbar \xi) \gamma \\
& =\sum_{(x, \xi) \in \Lambda}\left\langle f, \widehat{\mathcal{T}}^{\hbar}\left(h^{1 / 2} x, h^{1 / 2} \xi\right) \widehat{D}_{h^{-1 / 2}} g\right\rangle \widehat{\mathcal{T}}^{\hbar}(x, h \xi) \gamma \\
& =\sum_{\left(x^{\prime}, \xi^{\prime}\right) \in h^{1 / 2} \Lambda}\left\langle f, \widehat{\mathcal{T}}^{\hbar}\left(x^{\prime}, \xi^{\prime}\right) g^{h}\right\rangle \widehat{\mathcal{T}}^{\hbar}\left(h^{-1 / 2} x^{\prime}, h^{1 / 2} \xi^{\prime}\right) \gamma
\end{aligned}
$$

with unconditional convergence in $M_{m}^{p, q}\left(\mathbb{R}^{n}\right)$. Now, applying the isomorphism $\widehat{D}_{h^{-1 / 2}}$ to both sides of the previous equalities and exploiting the covariance property of $\widehat{\mathcal{T}}^{\hbar}(z)$ again, we obtain (28), that is $S_{\gamma, g}^{\hbar}=I$ on $M_{m}^{p, q, \hbar}\left(\mathbb{R}^{n}\right)$.

Since $g \in M_{v}^{1}$, it was proved in [20, Theorem 12.2.3] that the analysis operator $C_{g}$ is continuous from $M_{m}^{p, q}\left(\mathbb{R}^{n}\right)$ into $\ell_{m}^{p, q}(\Lambda)$. Using similar arguments as before we infer that the following diagram is commutative:

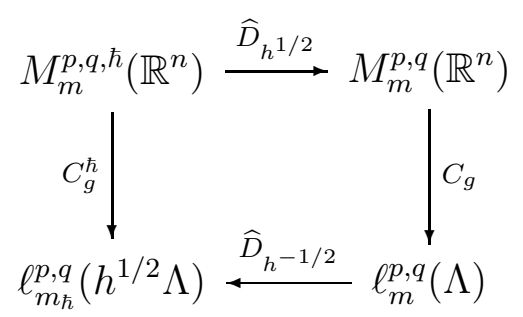

Notice that the lattice related to $C_{g}^{\hbar}$ is rescaled by $h^{1 / 2}$, whereas the domain of the weight $m$ is always $\Lambda$, that is why we are introduced the notation $m_{\hbar}$. Indeed, for $z \in h^{1 / 2} \Lambda$, setting $z=h^{1 / 2} w$, we have $w \in \Lambda$ and the weight $m_{\hbar}(z)=$ $m\left(h^{-1 / 2} h^{1 / 2} w\right)=m(w)$ does not depend on $h($ or $\hbar)$. We underline that

$$
\left\|C_{g}^{\hbar} f\right\|_{\ell_{m_{\hbar}}^{p, q}\left(h^{1 / 2} \Lambda\right)} \leq C\|f\|_{M_{m}^{p, q, \hbar}\left(\mathbb{R}^{n}\right)}, \quad f \in M_{m}^{p, q, \hbar}\left(\mathbb{R}^{n}\right)
$$

for suitable constant $C>0$ independent of $\hbar$. 
Arguing similarly we prove that also the diagram below is commutative:

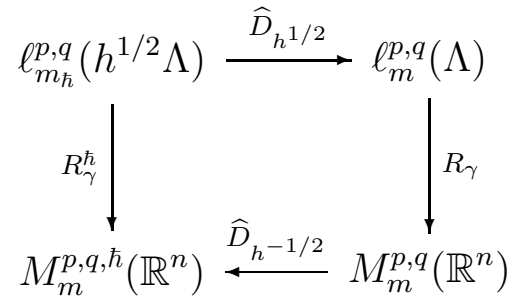

and we have

$$
\left\|R_{\gamma}^{\hbar} c\right\|_{M_{m}^{p, q, \hbar}\left(\mathbb{R}^{n}\right)} \leq C\|c\|_{\ell_{m_{\hbar}}^{p, q}\left(h^{1 / 2} \Lambda\right)}, \quad c \in \ell_{m_{\hbar}}^{p, q}\left(h^{1 / 2} \Lambda\right)
$$

for a positive constant $C$ independent of $\hbar$. From the continuity of the semi-classical analysis and synthesis operators we immediately obtain the norm equivalence (30). This concludes the proof.

\section{SEMI-CLASSICAL FIOS}

In this section we study the sparsity and continuity on $\hbar$-modulation spaces of a class of Fourier integral operators (FIOs) arising as propagators for certain variable coefficients Schrödinger equations. Precisely, we introduce the $\hbar$-version of the Wiener algebras of FIOs studied in [6] (see also [10]).

Consider a tame symplectomorphism $\chi: \mathbb{R}^{2 n} \rightarrow \mathbb{R}^{2 n}$, that is

(i) $\chi$ is smooth, invertible, and preserves the symplectic form in $\mathbb{R}^{2 n}$, i.e., $d x \wedge d \xi=$ $d y \wedge d \eta$, if $(x, \xi)=\chi(y, \eta)$;

(ii) $\chi$ satisfies

$$
\left|\partial_{z}^{\alpha} \chi(z)\right| \leq C_{\alpha} \quad|\alpha| \geq 1, z \in \mathbb{R}^{2 n} .
$$

Definition 3.1. Consider a $\hbar$-Gabor frame $\mathcal{G}^{\hbar}(g, \Lambda)$ with $g \in \mathcal{S}\left(\mathbb{R}^{n}\right), s \geq 0$, and $\chi$ be a tame symplectomorphism. We denote by $F I O_{\hbar}(\chi, s)$ the space of $\hbar$-dependent linear continuous operators $\widehat{A}^{\hbar}: \mathcal{S}\left(\mathbb{R}^{n}\right) \rightarrow \mathcal{S}^{\prime}\left(\mathbb{R}^{n}\right)$ such that

$$
\left|\left\langle\widehat{A}^{\hbar} \mathcal{T}^{\hbar}(z) g^{h}, \mathcal{T}^{\hbar}(w) g^{h}\right\rangle\right| \leq \frac{C_{s}}{v_{s}\left(h^{-1 / 2}(w-\chi(z))\right)}, \quad z, w \in \mathbb{R}^{2 n}
$$

for some constant $C_{s}>0$ independent of $\hbar$.

Observe that, for $\hbar=1 /(2 \pi)$ (hence $h=1$ ), we have $F I O_{1 /(2 \pi)}(\chi, s)=F I O(\chi, s)$, the class of FIOs studied in [6]. Using the formula

$$
\widehat{D}_{h^{-1 / 2}} \mathcal{T}(z) g=\mathcal{T}^{\hbar}\left(h^{1 / 2} z\right) g^{h}
$$

we see that $\widehat{A}^{\hbar}$ satisfies (32) if and only if $\widehat{B}^{\hbar}:=\widehat{D}_{h^{1 / 2}} \widehat{A}^{\hbar} \widehat{D}_{h^{-1 / 2}}$ satisfies

$$
\left|\left\langle\widehat{B}^{\hbar} \mathcal{T}(z) g, \mathcal{T}(w) g\right\rangle\right| \leq \frac{C_{s}}{v_{s}\left(w-\chi_{\hbar}(z)\right)}, \quad z, w \in \mathbb{R}^{2 n}
$$


for the same constant $C_{s}$, with

$$
\chi_{\hbar}(z)=\hbar^{-1 / 2} \chi\left(\hbar^{1 / 2} z\right) .
$$

We note that $\chi_{\hbar}$ satisfies, for every $\hbar \in(0,1]$, the same estimates (31) as $\chi$, with the same constants $C_{\alpha}$. Following the notation in [6], this means that $\widehat{B}^{\hbar} \in F \operatorname{FO}\left(\chi_{\hbar}, s\right)$.

Using the previous equivalence one can easily rephrase the main properties displayed by the class $F I O(\chi, s)$ in [6] to its semi-classical generalization $F I O_{\hbar}(\chi, s)$. We list the main features below. First, we present an equivalence between continuous decay conditions and the decay of the discrete $\hbar$-Gabor matrix for a linear operator $\widehat{A}^{\hbar}: \mathcal{S}\left(\mathbb{R}^{n}\right) \rightarrow \mathcal{S}^{\prime}\left(\mathbb{R}^{n}\right)$.

Theorem 3.2. Let $\widehat{A}^{\hbar}$ be a continuous linear operator $\mathcal{S}\left(\mathbb{R}^{n}\right) \rightarrow \mathcal{S}^{\prime}\left(\mathbb{R}^{n}\right)$ and $\chi$ a tame symplectomorphism. Consider a $\hbar$-Gabor frame $\mathcal{G}^{\hbar}(g, \Lambda)$ with $g \in \mathcal{S}\left(\mathbb{R}^{n}\right)$ and $s \geq 0$. Then the following properties are equivalent.

(i) There exists $C_{s}>0$ independent of $\hbar$ such that (32) holds true.

(ii) There exists $C_{s}>0$ independent of $\hbar$ such that

$$
\left|\left\langle\widehat{A}^{\hbar} \mathcal{T}^{\hbar}(\lambda) g^{h}, \mathcal{T}^{\hbar}(\mu) g^{h}\right\rangle\right| \leq \frac{C_{s}}{v_{s}\left(h^{-1 / 2}(\mu-\chi(\lambda))\right)}, \quad \lambda, \mu \in \Lambda .
$$

We call the infinite matrix $\left\{\left\langle\widehat{A}^{\hbar} \mathcal{T}^{\hbar}(\lambda) g^{h}, \mathcal{T}^{\hbar}(\mu) g^{h}\right\rangle\right\}_{\mu, \lambda \in \Lambda}$, the $\hbar$-Gabor matrix of the operator $\widehat{A}^{\hbar}$.

Using similar arguments to [6, Lemma 3.3] we obtain:

Lemma 3.3. The definition of $F I O_{\hbar}(\chi, s)$ is independent of the $\hbar$-Gabor frame $\mathcal{G}^{\hbar}(g, \Lambda)$.

3.1. Continuity properties and sparsity. The FIOs in $F I O_{\hbar}(\chi, s)$ display continuity results on semi-classical modulation spaces, with norms independent of $\hbar$, as explained in what follows.

Theorem 3.4. Consider $s>2 n, m \in \mathcal{M}_{v_{r}}$, with $0 \leq r<s-2 n$. Let $\widehat{A}^{\hbar} \in$ $F I O_{\hbar}(\chi, s)$. Then $\widehat{A}^{\hbar}$ extends to a bounded operator from $M_{m \circ \chi}^{p, \hbar}\left(\mathbb{R}^{n}\right)$ to $M_{m}^{p, \hbar}\left(\mathbb{R}^{n}\right)$, for $1 \leq p \leq \infty$, with operator norm uniformly bounded with respect to $\hbar$.

Proof. Since the operator $\widehat{B}^{\hbar}$ in (33) satisfies $\widehat{B}^{\hbar} \in F I O(\chi, s)$, we can apply to $\widehat{B}^{\hbar}$ the continuity results proved in [10, Theorem 4.1]. Precisely, an inspection of the proof of [10, Theorem 4.1] shows that such an operator $\widehat{B}^{\hbar}$ is bounded from $M_{m \circ \chi}^{p}\left(\mathbb{R}^{n}\right)$ into $M_{m}^{p}\left(\mathbb{R}^{n}\right)$ for every $1 \leq p \leq \infty$. Moreover its operator norm is uniformly bounded with respect to $\hbar$, because the constant $C_{s}$ in (33) is independent of $\hbar$, and the estimates (31) for $\chi_{\hbar}$ hold, as already observed, uniformly with respect to $\hbar$. By the very definition of the space $M_{m}^{p, \hbar}\left(\mathbb{R}^{n}\right)$ we easily attain the result.

Due to the bilipschitz property of $\chi, v_{r} \circ \chi \asymp v_{r}$, hence the previous result for polynomial weights $v_{r}$ rewrites as follows. 
Corollary 3.5. Consider $\widehat{A}^{\hbar} \in F I O_{\hbar}(\chi, s)$ and $0 \leq r<s-2 n$. Then $\widehat{A}^{\hbar}$ extends to a bounded operator on $M_{v_{r}}^{p, \hbar}\left(\mathbb{R}^{n}\right)$, for $1 \leq p \leq \infty$, with operator norm uniformly bounded with respect to $\hbar$.

For $p=2$, we have $M_{v_{r}}^{2}\left(\mathbb{R}^{n}\right)=\mathcal{Q}_{r}$, the Shubin-Sobolev spaces [2, 29]. Hence the previous issues hold also for the semi-classical version $\mathcal{Q}_{r}^{\hbar}:=M_{v_{r}}^{2, \hbar}\left(\mathbb{R}^{n}\right)$ of ShubinSobolev spaces.

Given a tame symplectomorphism $\chi$, let us define

$$
F I O_{\hbar}(\chi)=\cap_{s \geq 0} F I O_{\hbar}(\chi, s) .
$$

If $\widehat{A}^{\hbar} \in F I O_{\hbar}(\chi)$, then it satisfies (34) for every $s \geq 0$. This means the operator $\widehat{B}^{\hbar}$ in (33) has a Gabor matrix highly concentrated along the graph of the map $\chi_{\hbar}$, or equivalently, the $\hbar$-Gabor matrix of $\widehat{A}^{\hbar}$ is concentrated along the graph of the map $\chi$. This matrix property is called sparsity (cf. [11]) and using Theorem 3.4 we can state

Proposition 3.6. Any $\hbar$-dependent operator $\widehat{A}^{\hbar} \in F I O_{\hbar}(\chi)$ is bounded on $M_{v_{r}}^{p, \hbar}\left(\mathbb{R}^{n}\right)$ for every $1 \leq p \leq \infty, r \in \mathbb{R}$, with operator norm uniformly bounded with respect to $\hbar$.

3.2. Applications to Schrödinger propagators. We can now consider an application to the sparsity and continuity in modulation spaces of Schrödinger propagators.

We are interested in the Cauchy problem (8) with symbol $H(t, z), z=(x, \xi) \in$ $\mathbb{R}^{2 n}$, satisfying the Assumption $(\mathbf{H})$. Now, denote by $\chi^{(t, s)}, t, s \in[0, T]$, the Hamiltonian flow with Hamiltonian function $H$, i.e. $\left(x_{t, s}, \xi_{t, s}\right)=\chi^{(t, s)}(x, \xi)$ satisfies

$$
\dot{x}_{t, s}=\partial_{\xi} H\left(t, x_{t, s}, \xi_{t, s}\right), \quad \dot{\xi}_{t, s}=-\partial_{x} H\left(t, x_{t, s}, \xi_{t, s}\right)
$$

with initial value $\left(x_{s, s}, \xi_{s, s}\right)=(x, \xi)$ (the dot denoting the derivative with respect to $t)$. It is easily seen that the map $\chi^{(t, s)}$ satisfies estimates of the type (31) with constants $C_{\alpha}$ independent of $s, t \in[0, T]$.

Let finally $U(t, s)$ be the propagator for the equation in (8) , so that $U(s, s)=I$. We have the following result.

Proposition 3.7. The propagator $U(t, s)$ belongs to $F I O_{\hbar}\left(\chi^{(t, s)}\right)$ uniformly with respect to $t, s \in[0, T]$. As a consequence $U(t, s)$ is bounded on $M_{v_{s}}^{p, \hbar}\left(\mathbb{R}^{n}\right)$ for every $1 \leq p \leq \infty, s \in \mathbb{R}$, with operator norm uniformly bounded with respect to $\hbar \in(0,1]$, $s, t \in[0, T]$.

Proof. Consider the propagator $\tilde{U}(t, s)$ satisfying

$$
i \partial_{t} \tilde{U}(t, s)=\mathrm{Op}_{1}^{w}\left[\hbar^{-1} H\left(t, \hbar^{1 / 2} x, \hbar^{1 / 2} \xi\right)\right] \tilde{U}(t, s), \quad \tilde{U}(s, s)=I,
$$


and compare it with $U(t, s)$, which satisfies

$$
i \hbar \partial_{t} U(t, s)=\widehat{H(t, \cdot)} U(t, s) \quad U(s, s)=I .
$$

The two are related by the formula

$$
U(t, s)=\widehat{D}_{\hbar^{-1 / 2}} \tilde{U}(t, s) \widehat{D}_{\hbar^{1 / 2}} .
$$

Indeed we have

$$
\begin{aligned}
i \hbar \partial_{t} U(t, s) & =i \hbar \partial_{t} \widehat{D}_{\hbar^{-1 / 2}} \tilde{U}(t, s) \widehat{D}_{\hbar^{1 / 2}} \\
& =\widehat{D}_{\hbar^{-1 / 2}} \mathrm{Op}_{1}^{w}\left[H\left(t, \hbar^{1 / 2} x, \hbar^{1 / 2} \xi\right)\right] \tilde{U}(t, s) \widehat{D}_{\hbar^{1 / 2}} \\
& =\mathrm{Op}_{1}^{w}\left[H\left(t, x, \hbar_{\xi}\right)\right] \widehat{D}_{\hbar^{-1 / 2}} \tilde{U}(t, s) \widehat{D}_{\hbar^{1 / 2}} \\
& =\mathrm{Op}_{1}^{w}[H(t, x, \hbar \xi)] U(t, s) \\
& =\widehat{H(t, \cdot)} U(t, s) .
\end{aligned}
$$

Now, it was proved in [30, Corollary 7.4] that $\tilde{U}(t, s)$ satisfies an estimate of the form (33), namely

$$
|\langle\tilde{U}(t, s) \mathcal{T}(z) g, \mathcal{T}(w) g\rangle| \leq \frac{C_{n}}{v_{n}\left(w-\chi_{\hbar}^{(t, s)}(z)\right)}, \quad z, w \in \mathbb{R}^{2 n}
$$

for every $n \in \mathbb{N}$, where

$$
\chi_{\hbar}^{(t, s)}(z)=\hbar^{-1 / 2} \chi^{(t, s)}\left(\hbar^{1 / 2} z\right)
$$

is the flow corresponding to the Hamiltonian

$$
\hbar^{-1} H\left(t, \hbar^{1 / 2} z\right) \text {. }
$$

As a consequence, $U(t, s) \in F I O_{\hbar}\left(\chi^{(t, s)}\right)$ uniformly with respect to $s, t \in[0, T]$ and the desired continuity result follows from Proposition 3.6 .

\section{A parametrix CONSTRUCtion FOR Schrödinger EQUATIOns}

One can construct approximate solutions to the problem (8) by the the following construction. Let us first consider the case when the initial datum in a Gaussian function, possibly translated and modulated. From now on we fix $s=0$ in (8) as initial time.

Consider the solution $z_{t}=\left(x_{t}, \xi_{t}\right)$ to the Hamiltonian system

$$
\dot{x}_{t}=\partial_{p} H\left(t, x_{t}, \xi_{t}\right), \quad \dot{\xi}_{t}=-\partial_{x} H\left(t, x_{t}, \xi_{t}\right)
$$

with initial value (at $t=0) z_{0}=\left(x_{0}, \xi_{0}\right)$. Let $\chi_{t}$ be the Hamiltonian flow defined by $H(t, z)$, hence

$$
z_{t}=\chi_{t}\left(z_{0}\right) .
$$


Define

$$
H_{z_{0}}^{(l)}(t, z)=\sum_{|\gamma|=l} \frac{1}{\gamma !} \partial_{z}^{\gamma} H\left(t, z_{t}\right) z^{\gamma}, \quad z \in \mathbb{R}^{2 n}, l \geq 2 .
$$

It is well-known that the solution to the corresponding operator Schrödinger equation with $\hbar=1$, namely

$$
i \partial_{t} \widehat{S}_{t}\left(z_{0}\right)=\mathrm{Op}_{1}^{w}\left[H_{z_{0}}^{(2)}(t)\right] \widehat{S}_{t}\left(z_{0}\right) \quad \widehat{S}_{0}\left(z_{0}\right)=I
$$

is a metaplectic operator $\widehat{S}_{t}\left(z_{0}\right)$ corresponding to the symplectic matrix $S_{t}\left(z_{0}\right)$ via the metaplectic representation [14, 3]. In fact $S_{t}\left(z_{0}\right)$ is the (linear) Hamiltonian flow of $H_{z_{0}}^{(2)}(t)$ and therefore, as a consequence of (9) , the entries of the matrix $S_{t}\left(z_{0}\right)$ are bounded functions of $t \in[0, T]$ and $z_{0} \in \mathbb{R}^{2 n}$.

Now, an asymptotic solution to (8) , modulo $O\left(\hbar^{(N+1) / 2}\right), N \in \mathbb{R}$, with initial value being the coherent state $\phi_{z_{0}}^{\hbar}$ :

$$
\left\{\begin{array}{l}
i \hbar \partial_{t} u=\widehat{H(t)} u \\
u(0)=\phi_{z_{0}}^{\hbar}
\end{array}\right.
$$

is provided by the so-called Gaussian beam (see, e.g., [1, Section 3])

$$
\phi_{z_{0}}^{\hbar, N}(x)=e^{\frac{i}{\hbar} \delta\left(t, z_{0}\right)} \widehat{\mathcal{T}}^{\hbar}\left(z_{t}\right) \widehat{D}_{\hbar^{-1 / 2}} \widehat{S}_{t}\left(z_{0}\right) \sum_{j=0}^{N} \hbar^{j / 2} b_{j}(t, x) \phi_{0}(x) .
$$

Here $b_{0}(t, x)=1$ and for $j>0, b_{j}(t, x)$ is a polynomial in $x$, having coefficients depending on $t, z_{0}$ which are bounded (for details, see [1, Section 3]). The symmetrized action $\delta$ is defined by

$$
\delta\left(t, z_{0}\right)=\int_{0}^{t}\left(\frac{1}{2} \sigma\left(z_{s}, \dot{z}_{s}\right)-H\left(s, z_{s}\right)\right) d s
$$

with $\sigma$ being the standard symplectic form.

The functions $\phi_{z_{0}}^{\hbar, N}$ turn out to be approximate solutions in the sense that

$$
\begin{gathered}
R_{z_{0}}^{(N)}(t, \cdot):=\left(i \hbar \partial_{t}-\widehat{H(t)}\right) \phi_{z_{0}}^{\hbar, N}(t, \cdot) \\
=-e^{\frac{i}{\hbar} \delta\left(t, z_{0}\right)} \widehat{\mathcal{T}}^{\hbar}\left(z_{t}\right) \widehat{D}_{\hbar^{-1 / 2}} \widehat{S}_{t}\left(z_{0}\right)\left(\sum_{\substack{l+k \geq N+3 \\
3 \leq l \leq N+2 \\
0 \leq k \leq N}} \hbar^{(l+k) / 2} \mathrm{Op}_{1}^{w}\left[H_{z_{0}}^{(l)}\left(t, S_{t}\left(z_{0}\right) z\right)\right] b_{k}(t, \cdot) \phi_{0}\right. \\
\left.+\sum_{k=0}^{N} \hbar^{(N+3+k) / 2} \mathrm{Op}_{1}^{w}\left[r_{z_{0}}^{(N+3)}\left(t, S_{t}\left(z_{0}\right) z\right)\right] b_{k}(t, \cdot) \phi_{0}\right)
\end{gathered}
$$


is $O\left(\hbar^{(N+3) / 2}\right)$, where we set

$$
r_{z_{0}}^{(N+3)}(t, z):=\frac{1}{(N+3) !} \sum_{|\gamma|=N+3} \int_{0}^{1} \partial_{z}^{\gamma} H\left(t, z_{t}+\theta \hbar^{1 / 2} z\right) z^{\gamma}(1-\theta)^{N+2} d \theta .
$$

In [1] it was in fact introduced a parametrix via Gabor frames, valid for arbitrary $L^{2}$ initial data. Such a parametrix, having the previous Gaussian beams as building blocks, is constructed as follows.

For $\alpha \beta<1, \Lambda=\alpha \mathbb{Z}^{n} \times \beta \mathbb{Z}^{n}$, consider the $\hbar$-Gabor frame $\mathcal{G}^{\hbar}\left(\phi_{0}^{\hbar}, h^{1 / 2} \Lambda\right)$. Let $\gamma_{0}^{h}$ be the dual window in $\mathcal{S}\left(\mathbb{R}^{n}\right)$ defined in (20). For $N \geq 0, t \in[0, T]$, the parametrix to (8) is defined by

$$
\left[U^{(N)}(t) f\right](t, \cdot)=\sum_{z_{0} \in h^{1 / 2} \Lambda}\left\langle f, \widehat{\mathcal{T}}^{\hbar}\left(z_{0}\right) \gamma_{0}^{h}\right\rangle \phi_{z_{0}}^{\hbar, N}(t, \cdot) .
$$

Observe that $U^{(N)}(0) f=f$.

This is our main result.

Theorem 4.1. Consider $s \in \mathbb{R}$ and the weight function $v_{s}$ defined in (21). Under the Assumption $(\mathbf{H})$ and with the above notation, there exists a constant $C=C(T)$ independent of $\hbar$ such that, for every $f \in M_{v_{s}}^{p, \hbar}\left(\mathbb{R}^{n}\right), 1 \leq p \leq \infty$,

$$
\left\|U^{(N)}(t) f\right\|_{M_{v_{s}}^{p, \hbar}\left(\mathbb{R}^{n}\right)} \leq C\|f\|_{M_{v_{s}}^{p, \hbar}\left(\mathbb{R}^{n}\right)} \quad \forall t \in[0, T]
$$

and

$$
\left\|\left(i \hbar \partial_{t}-\widehat{H(t)}\right) U^{(N)}(t) f\right\|_{M_{v s}^{p, \hbar}\left(\mathbb{R}^{n}\right)} \leq C \hbar^{(N+3) / 2}\|f\|_{M_{v_{s}}^{p, \hbar}\left(\mathbb{R}^{n}\right)} \quad \forall t \in[0, T] .
$$

If $U(t)$ denotes the exact propagator, for every $f \in M_{v_{s}}^{p, \hbar}\left(\mathbb{R}^{n}\right)$,

$$
\left\|\left(U^{(N)}(t)-U(t)\right) f\right\|_{M_{v_{s}}^{p, \hbar}\left(\mathbb{R}^{n}\right)} \leq C t \hbar^{(N+1) / 2}\|f\|_{M_{v_{s}}^{p, \hbar}\left(\mathbb{R}^{n}\right)} \quad \forall t \in[0, T] .
$$

The proof relies on the following lemmas.

Lemma 4.2. Consider $t \in[0, T], z_{0} \in \mathbb{R}^{2 n}$, and the metaplectic operator $\widehat{S}_{t}\left(z_{0}\right)$, solution to (41). Define

$$
\widehat{S}_{t}^{\hbar}\left(z_{0}\right)=\widehat{D}_{\hbar^{-1 / 2}} \widehat{S}_{t}\left(z_{0}\right) \widehat{D}_{\hbar^{1 / 2}}
$$

Then the metaplectic operator $\widehat{S}_{t}^{\hbar}$ is continuous from $\mathcal{S}^{\hbar}$ to $\mathcal{S}^{\hbar}$ with operator seminorms bounded with respect to $t \in[0, T], z_{0} \in \mathbb{R}^{2 n}$, and independent of $\hbar$.

Proof. It was already shown in the proof of Theorem 3.4 in 1 that metaplectic operators $\widehat{S}_{t}\left(z_{0}\right)$ are bounded $\mathcal{S}\left(\mathbb{R}^{n}\right) \rightarrow \mathcal{S}\left(\mathbb{R}^{n}\right)$, with the entries of the matrix $S_{t}\left(z_{0}\right)$ being bounded functions of $t \in[0, T]$ and $z_{0} \in \mathbb{R}^{2 n}$. Hence the Schwartz seminorms are bounded with respect to $t, z_{0}$. Using

$$
\widehat{S}_{t}^{\hbar}\left(z_{0}\right)=\widehat{D}_{\hbar^{-1 / 2}} \widehat{S}_{t}\left(z_{0}\right) \widehat{D}_{\hbar^{1 / 2}}=\widehat{D}_{h^{-1 / 2}} \widehat{D}_{(2 \pi)^{-1 / 2}} \widehat{S}_{t}\left(z_{0}\right) \widehat{D}_{(2 \pi)^{1 / 2}} \widehat{D}_{h^{1 / 2}}
$$


we immediately obtain the result.

Lemma 4.3. Let $\mathcal{B}$ be a fixed bounded subset of $\mathcal{S}^{\hbar}\left(\mathbb{R}^{n}\right)$ (as a Fréchet space with the seminorms in (24) ) and $g \in \mathcal{S}\left(\mathbb{R}^{n}\right)$. Then for every $N \geq 0$ there exists a positive constant $C_{N}$ independent of $\hbar$ such that

$$
\left|\left\langle f, \widehat{\mathcal{T}}^{\hbar}(z) g^{h}\right\rangle\right| \leq \frac{C_{N}}{v_{N}\left(h^{-1 / 2} z\right)}, \quad \forall z \in \mathbb{R}^{2 n}
$$

for every $f \in \mathcal{B}$.

Proof. Observe that the mapping $\widehat{D}_{h^{1 / 2}}: \mathcal{S}^{\hbar}\left(\mathbb{R}^{n}\right) \rightarrow \mathcal{S}\left(\mathbb{R}^{n}\right)$ is an isomorphism. Hence, for $f \in \mathcal{B} \subset \mathcal{S}^{\hbar}\left(\mathbb{R}^{n}\right), \widehat{D}_{h^{1 / 2}} f$ belongs to a bounded subset of $\mathcal{S}\left(\mathbb{R}^{n}\right)$. As a consequence, for all $N \geq 0$, there exists $C_{N}>0$ (independent of $\hbar$ ) such that

$$
\left|\left\langle\widehat{D}_{h^{1 / 2}} f, \widehat{\mathcal{T}}(z) g\right\rangle\right|=\left|V_{g}\left(\widehat{D}_{h^{1 / 2}} f\right)(z)\right| \leq C_{N} v_{-N}(z), \quad z \in \mathbb{R}^{2 n}
$$

because $V_{g}: \mathcal{S}\left(\mathbb{R}^{n}\right) \rightarrow \mathcal{S}\left(\mathbb{R}^{2 n}\right)$ is continuous (see e.g., [20, Theorem 11.2.5]).

Now

$$
\left\langle\widehat{D}_{h^{1 / 2}} f, \widehat{\mathcal{T}}(z) g\right\rangle=\left\langle f, \widehat{\mathcal{T}}^{\hbar}\left(h^{1 / 2} z\right) g^{h}\right\rangle
$$

and the claim follows.

We now are ready to prove Theorem 4.1,

Proof of Theorem 4.1. Let us first prove (48). We consider the case $p<\infty$, and we leave to the reader the case $p=\infty$, which is completely similar. For $f \in M_{v_{s}}^{p, \hbar}\left(\mathbb{R}^{n}\right)$ we have, using (30) with $g^{h}=\gamma_{0}^{h}$ defined in (20),

$$
\begin{aligned}
\left\|U^{(N)}(t) f\right\|_{M_{v s}^{p, \hbar}}^{p} & =\sum_{w \in h^{1 / 2} \Lambda}\left|\left\langle U^{(N)}(t) f, \widehat{\mathcal{T}}^{\hbar}(w) \gamma_{0}^{h}\right\rangle\right|^{p} v_{s}\left(h^{-1 / 2} w\right)^{p} \\
& =\sum_{w \in h^{1 / 2} \Lambda}\left|\left\langle\sum_{z_{0} \in h^{1 / 2} \Lambda}\left\langle f, \widehat{\mathcal{T}}^{\hbar}\left(z_{0}\right) \gamma_{0}^{h}\right\rangle \phi_{z_{0}}^{\hbar, N}(t, \cdot), \widehat{\mathcal{T}}^{\hbar}(w) \gamma_{0}^{h}\right\rangle\right|^{p} v_{s}\left(h^{-1 / 2} w\right)^{p} \\
& \leq \sum_{w \in h^{1 / 2} \Lambda}\left(\sum_{z_{0} \in h^{1 / 2} \Lambda}\left|\left\langle f, \widehat{\mathcal{T}}^{\hbar}\left(z_{0}\right) \gamma_{0}^{h}\right\rangle\right|\left|\left\langle\phi_{z_{0}}^{\hbar, N}(t, \cdot), \widehat{\mathcal{T}}^{\hbar}(w) \gamma_{0}^{h}\right\rangle\right|\right)^{p} v_{s}\left(h^{-1 / 2} w\right)^{p} .
\end{aligned}
$$


Now,

$$
\begin{aligned}
\left|\left\langle\phi_{z_{0}}^{\hbar, N}(t, \cdot), \widehat{\mathcal{T}}^{\hbar}(w) \gamma_{0}^{h}\right\rangle\right| & \leq \sum_{j=0}^{N} \hbar^{j / 2}\left|\left\langle\widehat{\mathcal{T}}^{\hbar}\left(z_{t}\right) \widehat{D}_{\hbar^{-1 / 2}} \widehat{S}_{t}\left(z_{0}\right) b_{j}(t, \cdot) \phi_{0}, \widehat{\mathcal{T}}^{\hbar}(w) \gamma_{0}^{h}\right\rangle\right| \\
& =\sum_{j=0}^{N} \hbar^{j / 2}\left|\left\langle\widehat{D}_{\hbar^{-1 / 2}} \widehat{S}_{t}\left(z_{0}\right) b_{j}(t, \cdot) \phi_{0}, \widehat{\mathcal{T}}^{\hbar}\left(w-z_{t}\right) \gamma_{0}^{h}\right\rangle\right| \\
& =\sum_{j=0}^{N} \hbar^{j / 2}\left|\left\langle\widehat{S}_{t}^{\hbar}\left(z_{0}\right) b_{j}^{h}\left(t,(2 \pi)^{1 / 2} \cdot\right) \psi_{0}^{h}, \widehat{\mathcal{T}}^{\hbar}\left(w-z_{t}\right) \gamma_{0}^{h}\right\rangle\right|
\end{aligned}
$$

Observe that $b_{j}\left(t,(2 \pi)^{1 / 2} \cdot\right) \psi_{0} \in \mathcal{S}\left(\mathbb{R}^{n}\right)$ for every $j=0, \ldots, N$ and so $b_{j}^{h}\left(t,(2 \pi)^{1 / 2} \cdot\right) \psi_{0}^{h} \in$ $\mathcal{S}^{\hbar}\left(\mathbb{R}^{n}\right)$ with seminorms independent of $h$. Using Lemma 4.2 we have

$$
\widehat{S}_{t}^{\hbar}\left(z_{0}\right) b_{j}^{h}\left(t,(2 \pi)^{1 / 2} \cdot\right) \psi_{0}^{h} \in \mathcal{S}^{\hbar}\left(\mathbb{R}^{n}\right)
$$

with seminorms uniformly bounded with respect to $h$. Hence, by Lemma 4.3 we obtain

$$
\left|\left\langle\widehat{S}_{t}^{\hbar}\left(z_{0}\right) b_{j}^{h}\left(t,(2 \pi)^{1 / 2} \cdot\right) \psi_{0}^{h}, \widehat{\mathcal{T}}^{\hbar}\left(w-z_{t}\right) \gamma_{0}^{h}\right\rangle\right| \leq \frac{C_{\tilde{N}}}{v_{\tilde{N}}\left(h^{-1 / 2}\left(w-z_{t}\right)\right)}, \quad \forall z_{0}, w \in \mathbb{R}^{2 n}
$$

for a constant $C_{\tilde{N}}>0$ independent of $\hbar$.

These estimates yield, for every $\tilde{N} \geq 0$,

$$
\left|\left\langle\phi_{z_{0}}^{\hbar, N}(t, \cdot), \widehat{\mathcal{T}}^{\hbar}(w) \gamma_{0}^{h}\right\rangle\right| \leq \frac{C_{\tilde{N}, N}}{v_{\tilde{N}}\left(h^{-1 / 2}\left(w-z_{t}\right)\right)}
$$

for a convenient $C_{\tilde{N}, N}>0$ independent of $\hbar$.

Now, by the second inequality in (30) (again with $g^{h}=\gamma_{0}^{h}$ ) we have

$$
\sum_{z_{0} \in h^{1 / 2} \Lambda}\left|\left\langle f, \widehat{\mathcal{T}}^{\hbar}\left(z_{0}\right) \gamma_{0}^{h}\right\rangle\right|^{p} v_{s}\left(h^{-1 / 2} z\right)^{p} \leq C\|f\|_{M_{v s}^{p, \hbar}\left(\mathbb{R}^{n}\right)}^{p}
$$

for a suitable $C>0$. Using the $v_{|s|}$ moderateness of $v_{s}$, we can write $v_{s}(w) \leq$ $C v_{|s|}\left(w-z_{t}\right) v_{s}\left(z_{t}\right)$ for a convenient $C>0$. Easily follows from the assumption (9) (see e.g. [6]) that the map $z_{0} \rightarrow z_{t}$ is globally Lipschitz continuous and allows the equivalence

$$
v_{s}\left(z_{t}\right) \asymp v_{s}\left(z_{0}\right) \quad \forall z_{0} \in \mathbb{R}^{2 n}, \forall t \in \mathbb{R} .
$$

Hence (48) will follow by combining (53), (54) and (55), provided that the kernel

$$
K\left(w, z_{0}\right):=C_{\tilde{N}, N} v_{-\tilde{N}+|s|}\left(h^{-1 / 2}\left(w-z_{t}\right)\right) \asymp C_{\tilde{N}, N} v_{-\tilde{N}+|s|}\left(h^{-1 / 2}\left(\chi_{t}^{-1}(w)-z_{0}\right)\right)
$$


(the equivalence is due to the bi-Lipschitz property of the Hamiltonian flow $z_{0} \rightarrow$ $\chi_{t}\left(z_{0}\right)=z_{t}$ defined in (39) $)$ satisfies Schur's estimates

$$
\sup _{w \in h^{1 / 2} \Lambda} \sum_{z_{0} \in h^{1 / 2} \Lambda}\left|K\left(w, z_{0}\right)\right|<A_{1}, \quad \sup _{z_{0} \in h^{1 / 2} \Lambda} \sum_{w \in h^{1 / 2} \Lambda}\left|K\left(w, z_{0}\right)\right|<A_{2},
$$

with constants $A_{1}, A_{2}>0$ independent of $\hbar$. This is clearly the case if $\tilde{N}>2 n+|s|$ by the very definition (56). For example, making the change of variables $z^{\prime}=$ $h^{-1 / 2} z_{0}-h^{-1 / 2} \chi_{t}^{-1}(w)$, we infer

$$
\begin{aligned}
\sum_{z_{0} \in h^{1 / 2} \Lambda}\left|K\left(w, z_{0}\right)\right| & \lesssim \sum_{z_{0} \in h^{1 / 2} \Lambda} v_{-\tilde{N}+|s|}\left(h^{-1 / 2}\left(\chi_{t}^{-1}(w)-z_{0}\right)\right) \\
& =\sum_{z^{\prime} \in \Lambda-h^{-1 / 2} \chi_{t}^{-1}(w)} v_{-\tilde{N}+|s|}\left(z^{\prime}\right)<A_{1}
\end{aligned}
$$

for a constant $A_{1}>0$ independent of $w, h$ and $t$.

The proof of (49) is similar: by the same argument as in (53) we obtain

$$
\begin{aligned}
\|\left(i \hbar \partial_{t}-\widehat{H(t)}\right) & U^{(N)}(t) f \|_{M_{v s}^{p, \hbar}\left(\mathbb{R}^{n}\right)}^{p} \\
\leq & \sum_{w \in h^{1 / 2} \Lambda}\left(\sum_{z_{0} \in h^{1 / 2} \Lambda}\left|\left\langle f, \widehat{\mathcal{T}}^{\hbar}\left(z_{0}\right) \gamma_{0}^{h}\right\rangle\right|\left|\left\langle R_{z_{0}}^{(N)}(t, \cdot), \widehat{\mathcal{T}}^{\hbar}(w) \gamma_{0}^{h}\right\rangle\right|\right)^{p} v_{s}\left(h^{-1 / 2} w\right)^{p} .
\end{aligned}
$$

where $R_{z_{0}}^{(N)}(t, \cdot)$ is defined in (45); observe that in (45) we can take out the factor $\hbar^{(N+3) / 2}$ which appears in (49). The pseudodifferential operators appearing in (45) are easily treated observing that, by (9), their symbols together with their derivatives of every order are dominated by $C\langle z\rangle^{N+3}$, with a constant $C$ independent of $t \in[0, T]$ and $z \in \mathbb{R}^{2 n}$, so that they are continuous on $\mathcal{S}\left(\mathbb{R}^{n}\right)$ with operator seminorms uniformly bounded. The proof therefore goes on as that of (48).

Finally let us prove the formula (50). We use Duhamel's formula: if $U(t, s)$ is the exact propagator, with $U(s, s)=I$, and $U(t)=U(t, 0)$, we have

$$
U^{(N)}(t) f-U(t) f=-\frac{i}{\hbar} \int_{0}^{t} U(t, s)\left(i \hbar \partial_{s}-\widehat{H(s)}\right) U^{(N)}(s) f d s .
$$

Now the desired result follows at once from (49) and Minkowski inequality, using the fact that $U(t, s)$ is bounded $M_{v_{s}}^{p, \hbar}\left(\mathbb{R}^{n}\right) \rightarrow M_{v_{s}}^{p, \hbar}\left(\mathbb{R}^{n}\right)$ with norm uniformly bounded with respect to $\hbar, t, s$, when $0<\hbar \leq 1,0 \leq s \leq t \leq T$. This was in fact proved in Proposition 3.7.

Remark 4.4. (i) Observe that the results of the previous theorem hold true if we replace $v_{s}$ with a radial weight $m \in \mathcal{M}_{v_{s}}$. Indeed, we need that $m\left(z_{t}\right) \asymp m\left(z_{0}\right)$, for every $z_{0} \in \mathbb{R}^{2 n}$. 
(ii) Some technique (interchanging series) of this proof does not apply for $M_{m}^{p, q, \hbar}\left(\mathbb{R}^{n}\right)$, with $p \neq q$. In fact, the result does not hold for $M_{m}^{p, q, \hbar}\left(\mathbb{R}^{n}\right)$, with $p \neq q$ in general. A counterexample is provided by the quadratic Hamiltonian $H(x, \xi)=|x|^{2}$, $(x, \xi) \in \mathbb{R}^{2 n}$. In this case $U_{N}(t)=U(t)$ and the exact solution $U(t)$ is the multiplication operator $U(t) f(x)=e^{i|x|^{2}} f(x)$. It was proved in [10, Proposition 7.1] that this operator is not continuous on $M^{p, q}\left(\mathbb{R}^{n}\right)$, with $p \neq q$.

(iii) For $s \in \mathbb{R}, p=q=2$, recall that $M_{v_{s}}^{2}\left(\mathbb{R}^{n}\right)=\mathcal{Q}_{s}$, the Shubin-Sobolev spaces [2, 29]. Hence the results of the previous theorem hold also for semi-classical Shubin-Sobolev spaces.

\section{ACKNOWLEDGMENTS}

This research was partially supported by the Gruppo Nazionale per l'Analisi Matematica, la Probabilità e le loro Applicazioni (GNAMPA) of the Istituto Nazionale di Alta Matematica (INdAM), Italy.

\section{REFERENCES}

[1] M. Berra, I.M. Bulai, E. Cordero and F. Nicola. Gabor Frames of Gaussian Beams for the Schrödinger equation. Appl. Comput. Harmon. Anal., In Press. DOI: 10.1016/j.acha.2015.11.001

[2] P. Boggiatto, E. Cordero, and K. Gröchenig. Generalized Anti-Wick operators with symbols in distributional Sobolev spaces. Integral Equations and Operator Theory, 48(4):427-442, 2004.

[3] M. Combescure and D. Robert. Coherent states and applications in mathematical physics. Theoretical and Mathematical Physics. Springer, Dordrecht, 2012.

[4] M. Dimassi and J. Sjostrand, Spectral asymptotics in the semi-classical limit, Cambridge University Press, 1999.

[5] R. J. Duffin and A. C. Schaeffer. A class of nonharmonic fourier series. Trans. Amer. Math. Soc., 72:341-366, 1952.

[6] E. Cordero, K. Gröchenig, F. Nicola and L. Rodino. Wiener algebras of Fourier integral operators. J. Math. Pures Appl., 99:219-233, 2013.

[7] E. Cordero, K. Gröchenig, F. Nicola and L. Rodino. Generalized Metaplectic Operators and the Schrödinger Equation with a Potential in the Sjöstrand Class, J. Math. Phys., 55(8), art. no. $081506,2014$.

[8] E. Cordero and F. Nicola. Boundedness of Schrödinger Type Propagators on Modulation Spaces. J. Fourier Anal. Appl., 16:311-339, 2010.

[9] E. Cordero, F. Nicola and L. Rodino. Propagation of the Gabor Wave Front Set for Schrödinger Equations with non-smooth potentials. Rev. Math. Phys., 27 (1) art. no. 1550001, 2015.

[10] E. Cordero, F. Nicola and L. Rodino. Time-frequency analysis of Fourier integral operators. Commun. Pure Appl. Anal., 9(1):1-21, 2010.

[11] E. Cordero, F. Nicola and L. Rodino. Sparsity of Gabor representation of Schrödinger propagators. Appl. Comput. Harmon. Anal., 26(3):357-370, 2009.

[12] E. Cordero, F. Nicola, L. Rodino. Schrödinger equation with rough Hamiltonians. Discrete Contin. Dyn. Syst. Ser. A, 35(10):4805-4821, 2015. 
[13] I. Daubechies, A. Grossmann and Y. Meyer. Painless nonorthogonal expansions. J. Math. Phys., 27:1271-1283 1986.

[14] M.A. de Gosson. Symplectic methods in harmonic analysis and in mathematical physics, volume 7 of Pseudo-Differential Operators. Theory and Applications. Birkhäuser/Springer Basel AG, Basel, 2011.

[15] M.A. de Gosson. Hamiltonian deformations of Gabor frames: first steps. Appl. Comput. Harmon. Anal., 38(2):196-221, 2015.

[16] R. Gilmore. Geometry of symmetrized states. Ann. Phys. (NY) 74, 391-463, 1972.

[17] H.G. Feichtinger, Modulation spaces on locally compact abelian groups, Technical Report, University Vienna, 1983, and also in Wavelets and Their Applications, M. Krishna, R. Radha, S. Thangavelu, editors, Allied Publishers, 99-140, 2003.

[18] H.G. Feichtinger and T. Strohmer (eds.). Gabor Analysis and Algorithms: Theory and Applications. Birkhuser Boston, Boston, 1998.

[19] H.G. Feichtinger and T. Strohmer (eds.). Advances in Gabor analysis. Applied and Numerical Harmonic Analysis. Birkhäuser Boston Inc., Boston, 2003.

[20] K. Gröchenig. Foundations of Time-Frequency Analysis. Applied and Numerical Harmonic Analysis, Birkhäuser, Boston, MA, 2001.

[21] K. Gröchenig and Y. Lyubarskii. Gabor frames with Hermite functions. C. R. Math. Acad. Sci. Paris, 344(3):157-162, 2007.

[22] G.A. Hagedorn. Semiclassical quantum mechanics. I. The $\hbar \rightarrow 0$ limit for coherent states. Commun. Math. Phys., 71(1):77-93, 1980.

[23] G.A. Hagedorn. Semiclassical quantum mechanics. III. The large order asymptotics and more general states. Ann. Physics, 135(1):58-70, 1981.

[24] Yu. I. Lyubarski. Frames in the Bargmann space of entire functions. In Entire and subharmonic functions, 167-180. Amer. Math. Soc., Providence, RI, 1992

[25] F. Nicola. Convergence in $L^{p}$ for Feynman path integrals. Adv. Math., 294:384-409, 2016.

[26] A.M. Perelomov. Coherent states for arbitrary Lie group. Commun. Math. Phys., 26:222-236, 1972.

[27] K. Seip. Density theorems for sampling and interpolation in the Bargmann-Fock space. I. J. Reine Angew. Math., 429:91-106,1992

[28] K. Seip. and R. Wallstén. Density theorems for sampling and interpolation in the BargmannFock space. II. J. Reine Angew. Math., 429:107-113, 1992.

[29] M.A. Shubin. Pseudodifferential operators and spectral theory. Springer-Verlag, Berlin, second edition, 2001.

[30] D. Tataru, Phase space transforms and microlocal analysis, in Phase Space Analysis of Partial Differential Equations (Pubbl. Cent. Ric. Mat. Ennio Giorgi, Scuola Norm. Sup., Pisa, 2004), Vol. II, pp. 505524. See http://math.berkeley.edu/ tataru/papers/phasespace.pdf

[31] M. Zworski. Semiclassical analysis, volume 138 of Graduate Studies in Mathematics. American Mathematical Society, Providence, RI, 2012. 
Università di Torino, Dipartimento di Matematica, via Carlo Alberto 10, 10123 TORINO, ITALY

E-mail address: elena.cordero@unito.it

University of Vienna, Faculty of Mathematics, Oskar-Morgenstern-Platz 1 A1090 Wien, Austria

E-mail address: maurice.de.gosson@univie.ac.at

Dipartimento di Scienze Matematiche, Politecnico di Torino, corso Duca degli Abruzzi 24, 10129 Torino, ItAly

E-mail address: fabio.nicola@polito.it 